\title{
What evidence exists on conservation actions to conserve insects? A protocol for a systematic map of literature reviews
}

\author{
Neal R. Haddaway ${ }^{1,2,3^{*}} \mathbb{0}$, Eliza M. Grames ${ }^{4}$, Douglas H. Boyes ${ }^{5}$, Manu E. Saunders ${ }^{6}$ and Nigel G. Taylor ${ }^{7}$
}

\begin{abstract}
Background: Insects play a central role in the functioning of terrestrial and freshwater ecosystems and contribute to a multitude of ecosystem services in managed and unmanaged systems Even local declines of insect abundance and richness can have enormous ecological and economic consequences. Evidence-informed conservation actions are essential to prevent potential cascading consequences of insect declines, and to help declining populations recover. Policy-makers rely on syntheses of primary research, such as reviews and meta-analyses, when making decisions about which conservation actions to implement. These evidence reviews vary in their quality, and do not necessarily synthesise the full range of scientific evidence found in the primary literature, which hampers decision-making. This evidence review map will identify, catalogue, and describe evidence reviews that investigate actions and policies to conserve insect biodiversity. This will help policy makers identify relevant reviews and researchers to identify synthesis gaps. It will also generate a list of conservation actions which can feed into future synthesis projects. We will produce an interactive database of evidence reviews, acting as a bibliography for policy-makers, curate a list of insect conservation actions that have been reviewed, and identify synthesis gaps for conservation actions that have not been reviewed.

Methods: We will search for evidence reviews across seven large, generic bibliographic databases, a database of environmental reviews, and five grey literature resources using a search string consisting of an insect substring, a biodiversity or population response substring, an evidence synthesis substring, and a conservation substring. The results will be deduplicated and then screened at title and abstract (concurrently) and full text levels against predefined inclusion criteria. We will initially perform consistency checking on a subset of records at each level to ensure the inclusion criteria are sufficiently clear and understood by multiple reviewers. We will extract a suite of descriptive meta-data from relevant reviews, including a description of the action and information on each review's focal taxa, biomes, and locations. If resources allow, we will apply the CEESAT critical appraisal tool for evidence reviews to assess validity of individual records and the evidence base as a whole. We will summarise our findings in an interactive database of reviews and other visualisations, including evidence atlases and heat maps (cross tabulations of the volume of evidence across two categorical variables). The findings of the evidence review map will support the identification of synthesis gaps and clusters that may warrant further attention through secondary research.
\end{abstract}

Keywords: Entomology, Management, Systematic review, Systematic map, Evidence mapping, Intervention

*Correspondence: neal_haddaway@hotmail.com

${ }^{3}$ Mercator Research Institute on Global Commons and Climate Change, Torgauer Str 19, Berlin, Germany

Full list of author information is available at the end of the article

\section{Background}

There is no doubt that many insects are in trouble. Numerous reports have documented declines in insect diversity [1], abundance [2-4], and biomass $[5,6]$ over

c) The Author(s) 2020. This article is licensed under a Creative Commons Attribution 4.0 International License, which permits use, sharing, adaptation, distribution and reproduction in any medium or format, as long as you give appropriate credit to the original author(s) and the source, provide a link to the Creative Commons licence, and indicate if changes were made. The images or other third party material in this article are included in the article's Creative Commons licence, unless indicated otherwise in a credit line to the material. If material is not included in the article's Creative Commons licence and your intended use is not permitted by statutory regulation or exceeds the permitted use, you will need to obtain permission directly from the copyright holder. To view a copy of this licence, visit http://creativeco mmons.org/licenses/by/4.0/. The Creative Commons Public Domain Dedication waiver (http://creativecommons.org/publicdomain/ zero/1.0/) applies to the data made available in this article, unless otherwise stated in a credit line to the data. 
the past century, from the Arctic [7], to the tropics [8]. Not all insects are declining; in some places, there have been increases in insect biodiversity over the past century or population trends show little change over time [9-11]. Reports of insect population trends, whether in decline or increasing, have not been without controversy; there have been substantial biases identified in analyses, reporting, and synthesis (e.g. [12-21]), making it difficult to estimate global rates of decline. Regardless of ecological and statistical challenges in determining global rates of decline, even local declines of insect abundance and richness are predicted to have enormous ecological and economic consequences.

Insects are central to life on earth. More than half of all described species are insects, and there are an estimated 5.5 million insect species on Earth [22, 23]. Insects are critical to ecosystem functioning and global food production, contributing to a multitude of ecosystem services in managed and unmanaged systems. Insects are key components of many food webs, supporting insectivorous species such as birds, bats, and amphibians, and the species which rely on insectivores [24]. Around 35\% of global food production benefits from insect pollination $[25,26]$. Insects also regulate terrestrial and freshwater populations of plants and animals through herbivory, predation, and parasitism, including serving as natural enemies and biological control agents for pest species $[27,28]$. Four main ecosystem services provided by insects have been valued at more than $\$ 57$ billion USD per year in the United States alone [29]. Globally, insect pollination services alone are estimated to be $\$ 518$ billion USD each year [30]; the full economic value provided by insects worldwide is unknown. Other ecosystem services provided by insects, such as nutrient cycling, decomposition, soil formation, and cultural services are more difficult to value monetarily [31]. If insects are declining at estimated rates of $1-2 \%$ per year $[2,6,32,33]$, the results could be catastrophic.

Evidence-informed conservation actions are essential to help declining insect populations recover and prevent potential cascading effects to other taxa. Many entomologists and conservation biologists have called for policies to mitigate the effects of insect decline and promote insect population recovery (e.g. [15, 34, 35]). Suggested and tested solutions include habitat restoration and protection, agri-environment schemes, reducing pesticide use, and mitigating threats from invasive species, among other policy changes [34]. Detailed insect conservation actions are described in the primary literature, however, policy-makers should ideally rely on evidence reviews, such as systematic reviews and meta-analyses, when making decisions [36-38]. There are already many syntheses of certain insect conservation actions (e.g.
[39-46]), but there are a multitude of possible insect conservation actions that may exist only in the primary literature and are inaccessible to policy-makers in the form of evidence reviews. In the fields of health and social policy, it is common to synthesise existing reviews $[47,48]$, and this approach may be particularly advantageous in the field of insect conservation.

In order to prioritise where syntheses of insect conservation actions are needed for policy recommendations, it is critical to know what syntheses exist, which conservation actions have been reviewed, and which synthesis gaps remain. Furthermore, such a map of evidence reviews is vital for facilitating access to robust reviews across insect conservation, and increasing the use of robust evidence in insect conservation research, policy and practice $[36,49,50]$.

\section{Objective of the review}

In this review we aim to identify, catalogue and describe evidence reviews that investigate actions and policies to conserve insect biodiversity. We aim to produce an interactive database of evidence reviews, which will provide (a) a bibliography of insect conservation reviews and a list of conservation actions, outcomes, biomes, and taxa therein, and (b) a resource for future quantitative and qualitative syntheses on the effectiveness of insect biodiversity conservation actions, as an important source of evidence for inclusion in rigorous systematic reviews. In the process of creating the database of existing reviews, we also aim to identify synthesis gaps and clusters, directing future synthesis effort to where it is most needed. The review sits within a broader project aiming to identify knowledge gaps related to insect conservation actions (https://insectconservation.github.io/).

This synthesis has the following review question: What evidence reviews exist in the research literature on the effects of insect biodiversity conservation actions, and what actions and systems do they describe?

We have deliberately used the term 'action' below, rather than intervention, which is the traditional key element as part of the PICO acronym. This is a very deliberate choice that was guided by a global stakeholder engagement process. Key stakeholders pointed out that the term 'intervention' was viewed very negatively in some social-environmental contexts, and the term 'action' was preferable. This is also in keeping with conservation traditions of other organisations and research groups (e.g. Conservation Evidence). The question can be broken down into the following key elements:

Population: Insects in any habitat and any geographic region globally.

Action: Any policy or management practice that can be applied to conserve insects. 
Desired outcome: An action should aim to improve or reduce the rate or extent of negative impacts on any measure of insect biodiversity.

Study type: Any evidence reviews in the academic literature (including academic grey literature) aiming to identify, collate, and describe or summarise primary research studies, including narrative reviews, systematic reviews, systematic maps, meta-analyses, and other forms of evidence synthesis.

\section{Methods}

This synthesis will follow the methodological guidance for 'evidence review maps' set out by O'Leary et al. [37], and conforms to ROSES reporting standards (see Additional file 1) [51].

\section{Stakeholder engagement and question formulation}

The question to be investigated was proposed by the Swedish research foundation Mistra, in an effort to support the identification and prioritisation of knowledge gaps that warrant primary research funding. As a result, the question and its scope were fixed by Mistra, however, we will engage with a diverse range of stakeholders in the development of the project to ensure the results of our evidence review map are useful to researchers and decision-makers. Stakeholders will be identified by consulting known experts in the field (based on their previous work) and asking for suggestions from their network that could help guide the project, especially non-academics from non-governmental organizations or government departments focused on insect conservation. The broader project described above will engage with a diverse range of stakeholders to support the development of an ontology of insect biodiversity conservation actions and validation of the emergent knowledge gaps. Stakeholders will be selected from a related project undertaken to map stakeholders interested in insect conservation (EntoGEM; https://entogem.github.io/).

\section{Searching for articles Bibliographic databases}

We will search across seven large, multidisciplinary bibliographic databases and the CEEDER database of environmental reviews (Table 1). The results of searches from each database will be downloaded and then assembled and deduplicated in R 4.0.0 [52] using the package synthesiser [53].

\section{Bibliographic search string}

We will use the following search string in searches across titles, abstracts and keywords in each database. The string consists of four substrings: an insect group substring, a biodiversity or population response substring, an evidence synthesis substring, and a conservation action substring (Table 2). There exist syntheses of conservation actions and biodiversity responses that would benefit insects and other invertebrates, but that are not specifically focused on insects [54]. This is a limitation of our search strategy, which is necessarily confined solely to insects due to the resources available for screening search results that cannot support a search as broad as all syntheses of conservation actions targeting any taxa.

\section{Assessing the comprehensiveness of the search}

We have tested the comprehensiveness of the search string (Table 2) by assessing whether reviews of known relevance were returned by searches in five of the bibliographic databases we are searching (Academic Search Premier, BIOSIS Citation Index, CAB Abstracts, Scopus, and Zoological Record). For any reviews that were not retrieved by our search, we identified which concept category was not being matched and which relevant terms from the title, abstract, and keywords of missing reviews should be added to our search string. Of our benchmark list of 46 reviews (Additional file 2), 37 were retrieved after adding "conserv"*", "habitat", "insect", and "insects" to the biodiversity and population response outcomes and "treatment" to the conservation actions substring. The remaining nine articles could not be retrieved by revising the search because they were not indexed in the databases used to test the search $[55,56]$, made no mention of insects or invertebrates in the title, abstract, or keywords [57-61], or had no indication of being a review in the title, abstract, or keywords $[62,63]$. Because these articles cannot be retrieved by revising the search string while maintaining our concept category substrings, we concluded that our bibliographic database search strategy is sufficient.

\section{Grey literature}

We will target grey literature by searching across Google Scholar, DART-Europe E-theses, ProQuest Dissertations \& Theses A\&I, the Networked Digital Library of Theses and Dissertations Global ETD Search, and OpenThesis. We will use litsearchr $[63,64]$ to search NDLTD Global ETD Search and OpenThesis with the full search string and will use simplified searches (see Table 1) for thesis databases that do not have the capacity to support the full search string. We will not search for grey literature on individual organisational websites, due to resource constraints, but also because relevant literature reviews are highly unlikely to be found here and not elsewhere.

Results will be downloaded and merged with bibliographic search results prior to deduplication where possible. Where results cannot be saved as a csv file, they will be downloaded using either Publish or Perish (www. 
Table 1 Bibliographic databases and grey literature sources to be searched, including platforms used to access databases and years of institutional subscription access, where applicable. Search string modifications for grey literature sources with limitations are defined

\begin{tabular}{|c|c|c|c|}
\hline Platform & Database & Years & Modifications to search string \\
\hline \multirow[t]{2}{*}{ EBSCO } & Academic Search Premier & $1887-2020$ & $\mathrm{n} / \mathrm{a}$ \\
\hline & CAB Abstracts & $1973-2020$ & \\
\hline \multirow[t]{9}{*}{ Web of Science } & BIOSIS Citation Index & $1926-2020$ & $\mathrm{n} / \mathrm{a}$ \\
\hline & Core collections & & \\
\hline & SCI-EXPANDED & $1945-2020$ & \\
\hline & SSCl & $1956-2020$ & \\
\hline & $\mathrm{A} \& \mathrm{HCl}$ & $1975-2020$ & \\
\hline & CPCl-S & 1990-2020 & \\
\hline & CPCl-SSH & 1990-2020 & \\
\hline & ESCl & $2015-2020$ & \\
\hline & Zoological Record & $1864-2020$ & \\
\hline Lens.org & $\begin{array}{l}\text { CORE } \\
\text { Crossref } \\
\text { PubMed } \\
\text { PubMed Central } \\
\text { Microsoft Academic }\end{array}$ & $\mathrm{n} / \mathrm{a}$ & $\begin{array}{l}\text { All substrings will be searched in title or abstract with proximity operator replaced with } \sim \mathrm{n} \\
\text { and restructured so that only one term will be within proximity to a set of terms. For } \\
\text { example, in the biodiversity or population response substring, the first paired combi- } \\
\text { nation will become ((densit* OR diverse OR diversit* OR richness OR abundance OR } \\
\text { composition OR similarit* OR distribution* OR occupancy) AND communit* } \sim 10 \text { ) and the } \\
\text { same will be repeated for species and population* }\end{array}$ \\
\hline Scopus & Scopus & $1788-2020$ & W/n used as proximity operator \\
\hline Google Scholar & Google Scholar & $\mathrm{N} / \mathrm{A}$ & $\begin{array}{l}\text { insect "systematic map" OR "systematic review" OR "evidence review" OR "evidence map" OR } \\
\text { "systematic literature review" OR metaanalysis OR "meta analysis" OR meta-analysis OR } \\
\text { "quantitative synthesis" }\end{array}$ \\
\hline DART-Europe & DART-Europe E-theses & $\mathrm{n} / \mathrm{a}$ & insect AND (review OR meta-analysis) \\
\hline ProQuest & Dissertations \& Theses A\&l & $1861-2020$ & noft(insect AND (review OR meta-analysis)) \\
\hline NDLTD & $\begin{array}{l}\text { Networked Digital Library } \\
\text { of Theses \& Dissertations }\end{array}$ & $1970-2020$ & $\mathrm{n} / \mathrm{a}$ \\
\hline OpenThesis & OpenThesis & $1602-2020$ & synthesis substring searched only in title and abstract; other substrings searched in full text \\
\hline CEEDER & CEEDER & 2018-2019 & insect OR invert* \\
\hline
\end{tabular}

harzing.com), the Grey Literature Search Recorder app (https://www.eshackathon.org/software/grey-lit-repor ter.html), litsearchr $[63,64]$, or custom functions in $\mathrm{R}$ to scrape records into a.bib or.ris format where possible. Otherwise, they will be screened in place (i.e. online) and relevant records will be exported.

All search strings for each database and grey literature source are provided in Additional file 3 along with documentation of changes that deviate from the primary search string due to database limitations or specifications.

\section{Search language}

Searches will be performed in English only, since the databases we are using catalogue records with Englishlanguage titles, abstracts and keywords. We recognise that probably one-third of the literature on biodiversity and conservation is published in languages other than English [65] and that evidence published in English is likely a biased sample [66]; however, resources for translation are not available within the scope of this project.
For any reviews published in languages other than English, we will make an effort to extract actions from English abstracts or article summaries and will catalogue them for future translation.

\section{Supplementary searches}

In order to supplement our searches above, we will also search for relevant reviews within the large database of records collated through the EntoGEM project [67]. This project has searched across a suite of bibliographic databases and other resources in order to identify studies and datasets documenting insect population and biodiversity trends globally. The EntoGEM project search string was more sensitive than our search string for insect taxa but more specific for population outcomes and study duration, resulting in approximately 143,000 unique records. Since the EntoGEM search covered a wider range of sources than directly considered for the current review, it is possible that it contains additional evidence syntheses. We will apply the evidence synthesis and conservation substrings listed above (see Bibliographic search string) 
Table 2 Full search string grouped by substrings representing our four concept categories of insects, biodiversity or population responses, evidence synthesis, and conservation action

\begin{tabular}{|c|c|}
\hline Substring & Search terms \\
\hline \multirow[t]{2}{*}{ Insects } & 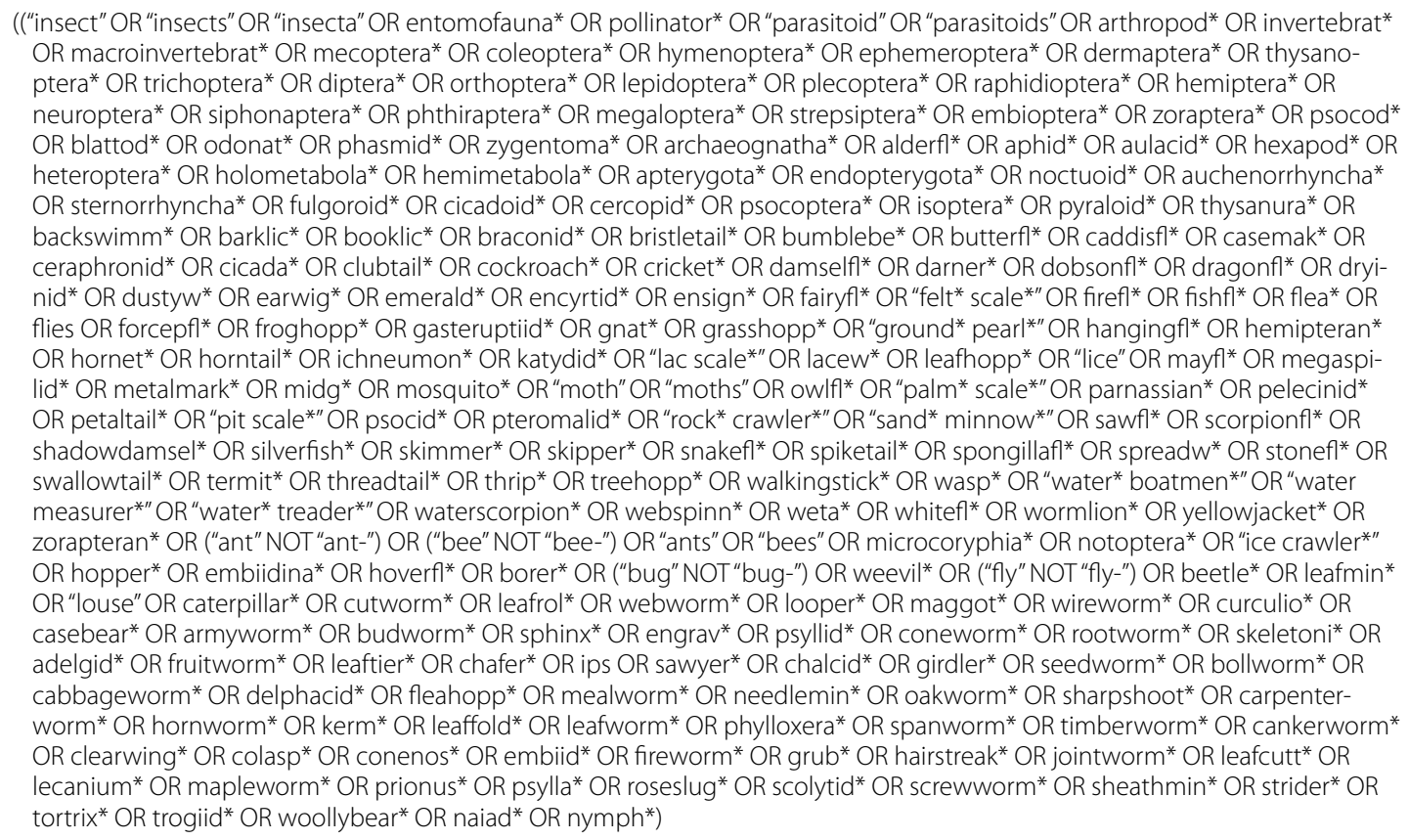 \\
\hline & AND \\
\hline \multirow[t]{2}{*}{$\begin{array}{l}\text { Biodiversity or } \\
\text { population } \\
\text { response }\end{array}$} & $\begin{array}{l}\text { (((communit* OR species OR population* OR "insect" OR "insects") NEAR/10 (densit* OR diverse OR diversit* OR richness OR } \\
\text { abundance OR composition OR similarit* OR distribution* OR occupancy OR conserv* OR habitat)) OR biodiversity OR "func- } \\
\text { tional diversity" OR "species loss" OR "loss* of species" OR "number of species" OR (species AND (focal OR target OR keystone OR } \\
\text { umbrella OR red-list* OR threatened OR endangered OR rare OR indicator))) }\end{array}$ \\
\hline & AND \\
\hline \multirow[t]{2}{*}{ Evidence synthesis } & $\begin{array}{l}\text { ((synthes* NEAR/5 (evidence OR research OR quantitative OR qualitative OR "mixed method*")) OR (systematic* NEAR/5 map*) OR } \\
\text { (evidence NEAR/5 map*) OR "meta-analys*" OR "meta analys*"OR review) }\end{array}$ \\
\hline & AND \\
\hline Conservation action & $\begin{array}{l}\text { (conserv* OR manag* OR restor* OR preserv* OR action* OR interven* OR policy OR policies OR practice* OR protect* OR reintro- } \\
\text { duc* OR scheme* OR regulat* OR legislat* OR "rule" OR "rules" OR subsidi* OR tarif* OR reform* OR "measure" OR "measures" OR } \\
\text { "treatment")) }\end{array}$ \\
\hline
\end{tabular}

to the EntoGEM search results to extract any potentially relevant syntheses. These will be added to the bibliographic search results, deduplicated, and screened as described above.

\section{Article screening and study eligibility criteria Screening process}

All bibliographic database results will be uploaded to a public project on SysRev.com for screening, following deduplication. Screening will be undertaken at title and abstract level (concurrently) and then at full text level. Intercoder reliability in syntheses typically uses the kappa statistic [68] to assess levels of agreement and error rates in the data, but kappa assumes similar error rates for all screeners and does not account for the true classification.
As an alternative to kappa, we will use false positive and false negative error rates to assess reliability. All coders will screen 619 studies in duplicate, which is the minimum number needed to assess if the calculated false negative error rate is below 0.10 with a Type II error rate of 0.80 and a Type I error rate of 0.05 . All disagreements will be resolved by discussion and consensus or by a third party. Resolved decisions on articles will be used to assess false positive and false negative error rates for all screeners. After assessing error rates, coders with false negative rates below 0.05 and false positive rates below 0.15 will screen articles singly. Any coders with error rates outside the acceptable bounds will continue screening in duplicate and error rates will be recalculated using a sliding window of the most recent 619 articles they have 
screened until error rates are within acceptable bounds. A list of all articles excluded at full text screening level with exclusion reasons will be provided along with the final review report.

\section{Eligibility criteria}

Records will be included in this review if they adhere to the following criteria.

Population: Insect biodiversity, including populations, communities, or species, in any habitat and any geographic region globally.

Action: Any policy or management practice applied to conserve insect biodiversity, including the maintenance of practices not specifically aimed at conserving insects that indirectly benefit insects, the reduction of harmful practices, and possible actions not yet widely applied for the purposes of insect conservation.

Outcome: Insect biodiversity, including: population abundance; presence of indicator, rare, or threatened species; species richness, diversity, and composition; or other measures reported to be a proxy or indicator of biodiversity. Biodiversity should be the intended outcomes of the intervention, but there does not have to be any synthesis of effects on these outcomes. For example, a synthesis could just list or map different insect conservation interventions used around the world without any synthesis of their effects.

Study type: Any evidence synthesis aiming to identify, collate, describe, and summarise primary empirical research studies. This includes: systematic reviews, metaanalyses, systematic maps, evidence and gap maps, rapid reviews, scoping reviews, and literature reviews using partially systematic methods. Authors of the synthesis must state clearly that it is a 'review' or 'meta-analysis' and must refer in some way to methods used to identify and collate the included literature, although we will include syntheses with an incomplete description of methods used. We will not include site-specific/ case-study syntheses unless they involve a review of the literature.

We will ensure that no reviewer screens records for any article that they have authored.

\section{Study validity assessment}

Since this synthesis aims only to map existing evidence syntheses, we do not intend to conduct an in-depth appraisal of the validity of included syntheses. However, if resources allow, we will use CEESAT $2.0[69,70]$ to assess the validity of included syntheses. Appraisal will begin with an initial consistency checking of application of the CEESAT 2.0 tool by two or more reviewers, with all disagreements discussed and criteria clarified prior to appraisal of the full set of included reviews. The results of this assessment would be used in narrative synthesis to summarise the numbers of studies with different ratings across the evidence base.

\section{Data coding strategy}

Two reviewers will extract the data listed in Table 3 from a preliminary set of 10 full text articles, discussing all discrepancies in coding and extracted meta-data before a single reviewer goes on to extract data from the remaining syntheses. We will extract the following information from included syntheses.

Where information is missing, we will attempt to contact the authors with a request for data. We will ensure

Table 3 Meta-data to be extracted from relevant reviews

\begin{tabular}{ll}
\hline Item & Description \\
\hline Title & Published title \\
Year & Year of publication \\
Authors & Author's records \\
Journal & Full journal name \\
Volume & Journal volume \\
Pages & Article pages \\
Abstract & Published summary text \\
Action name(s) & Short 1- to 3- word description of all actions included in a synthesis \\
Action description(s) & Authors'full description of the action(s) \\
Focal taxa/taxon & Stated taxa/taxon that is the focus of the action(s) \\
Focal biome & Biome in which the focal taxa/taxon exists, as it has been classified by the \\
& authors of the review. These biomes will be reclassified post hoc using \\
Focal geographical region & a standard ontology [54] \\
\hline
\end{tabular}


that no reviewer extracts data from any article that they have authored.

\section{Study mapping and presentation}

Meta-data extracted from relevant syntheses will be presented in an interactive, searchable database, accompanied by visualisations in the form of histograms, heat maps (cross tabulations of the number of studies across 2 categorical variables), and an evidence atlas (i.e., a choropleth map showing the number of syntheses across different regions of the world). A review report will narratively summarise the literature, including an appraisal of the validity of the individual syntheses and the evidence base as a whole if resources allow.

We will use the visualisations we produce to identify underrepresented actions, taxa, biomes and regions in the evidence base. We will list clear gaps (almost complete lack of reviews) where synthesis may be warranted. We will also highlight topics where syntheses exist of low validity (if critical appraisal is conducted) and where rigorous systematic review may be warranted.

\section{Supplementary information}

Supplementary information accompanies this paper at https://doi. org/10.1186/s13750-020-00214-8.

Additional file 1. ROSES form for systematic map protocols. This file holds the completed ROSES form for the systematic map protocol.

Additional file 2. Benchmark list of articles of known relevance for comprehensiveness testing. This file is a bibliography containing all articles used in benchmark testing of the search strings.

Additional file 3. Search string modifications. This file lists the full search strings used across all resources.

\section{Acknowledgements}

The authors thank Biljana Macura for discussions during planning of this project and Andrew Bladon, Chris Elphick, and Matt Forister for helpful feedback on earlier versions of this protocol.

\section{Authors' contributions}

$\mathrm{NRH}$ and EMG drafted the manuscript with edits from all authors. All authors read and approved the final manuscript.

\section{Funding}

Open Access funding provided by Stockholm University. This work was funded by Mistra (https://www.mistra.org/en).

\section{Availability of data and materials}

Not applicable.

\section{Ethics approval and consent to participate}

Not applicable.

\section{Consent for publication}

Not applicable.

\section{Competing interests}

The authors declare they have no competing interests.

\section{Author details}

1 Stockholm Environment Institute, Linnégatan 87D, Stockholm, Sweden.

${ }^{2}$ Africa Centre for Evidence, University of Johannesburg, Johannesburg, South Africa. ${ }^{3}$ Mercator Research Institute on Global Commons and Climate Change, Torgauer Str 19, Berlin, Germany. ${ }^{4}$ University of Connecticut, Storrs, CT, USA.

${ }^{5}$ UK Centre for Ecology \& Hydrology, Wallingford, UK. ${ }^{6}$ School of Environmental \& Rural Science, University of New England, Armidale, NSW, Australia. ${ }^{7}$ Tour du Valat, Research Institute for the Conservation of Mediterranean Wetlands, 13200 Arles, France.

Received: 29 May 2020 Accepted: 21 November 2020

Published online: 17 December 2020

\section{References}

1. Brooks DR, Bater JE, Clark SJ, Monteith DT, Andrews C, Corbett SJ, et al. Large carabid beetle declines in a United Kingdom monitoring network increases evidence for a widespread loss in insect biodiversity. J Appl Ecol. 2012:49(5):1009-19.

2. Hallmann CA, Zeegers T, van Klink R, Vermeulen R, van Wielink P, Spijkers $H$, et al. Declining abundance of beetles, moths and caddisflies in the Netherlands. Insect Conserv Divers. 2020;13(2):127-39.

3. Herrera CM. Complex long-term dynamics of pollinator abundance in undisturbed Mediterranean montane habitats over two decades. Ecol Monogr. 2019;89(1):e01338.

4. Schuch S, Bock J, Krause B, Wesche K, Schaefer M. Long-term population trends in three grassland insect groups: a comparative analysis of 1951 and 2009. J Appl Entomol. 2012;136(5):321-31.

5. Hallmann CA, Sorg M, Jongejans E, Siepel H, Hofland N, Schwan H, et al. More than 75 percent decline over 27 years in total flying insect biomass in protected areas. PLoS ONE. 2017;12(10):e0185809.

6. Wepprich T, Adrion JR, Ries L, Wiedmann J, Haddad NM. Butterfly abundance declines over 20 years of systematic monitoring in Ohio, USA. PLoS ONE. 2019:14(7):e0216270.

7. Loboda S, Savage J, Buddle CM, Schmidt NM, Høye TT. Declining diversity and abundance of High Arctic fly assemblages over two decades of rapid climate warming. Ecography. 2018;41(2):265-77.

8. Roubik DW. Ups and downs in pollinator populations: when is there a decline? Conserv Ecol. 2001;5(1):2.

9. Boyes DH, Fox R, Shortall CR, Whittaker R. Bucking the trend: the diversity of Anthropocene 'winners' among British moths. Front Biogeogr. 2019;11(3):e43862.

10. Crossley MS, Meier AR, Baldwin EM, Berry LL, Crenshaw LC, Hartman GL, et al. No net insect abundance and diversity declines across US Long Term Ecological Research sites. Nat Ecol Evol. 2020;4(10):1368-76.

11. van Klink R, Bowler DE, Gongalsky KB, Swengel AB, Gentile A, Chase JM. Meta-analysis reveals declines in terrestrial but increases in freshwater insect abundances. Science. 2020;368(6489):417-20.

12. Grames EM, Elphick CS. Use of study design principles would increase the reproducibility of reviews in conservation biology. Biol Cons. 2020;241:108385

13. Komonen A, Halme P, Kotiaho JS. Alarmist by bad design: Strongly popularized unsubstantiated claims undermine credibility of conservation science. Rethink Ecol. 2019:4:17-9.

14. Mupepele A-C, Bruelheide H, Dauber J, Krüß A, Potthast T, Wägele W, et al. Insect decline and its drivers: unsupported conclusions in a poorly performed meta-analysis on trends-A critique of Sánchez-Bayo and Wyckhuys. Basic Appl Ecol. 2019;37:20-3.

15. Saunders ME, Janes JK, O'Hanlon JC. Moving on from the insect apocalypse narrative: engaging with evidence-based insect conservation. Bioscience. 2020;70(1):80-9.

16. Schowalter TD, Willig MR, Presley SJ, Pandey M. Warnings of an "insect apocalypse" are premature. Front Ecol Environ. 2019;17(10):547.

17. Simmons BI, Balmford A, Bladon AJ, Christie AP, De Palma A, Dicks LV, et al Worldwide insect declines: an important message, but interpret with caution. Ecol Evol. 2019;9(7):3678-80.

18. Thomas $\mathrm{C}$, Jones TH, Hartley SE. "Insectageddon": a call for more robust data and rigorous analyses. Glob Change Biol. 2019;25(6):1891-2.

19. Wagner DL. Global insect decline: comments on Sánchez-Bayo and Wyckhuys. Biol Cons. 2019;233:332-3. 
20. Welti EAR, Joern A, ellison a, Lightfoot DC, Record S, Rodenhouse N, et al. Meta-analyses of insect temporal trends must account for the complex sampling histories inherent to many long-term monitoring efforts: EcoEvoRxiv; 2020 2020/08//.

21. Willig MR, Woolbright L, Presley SJ, Schowalter TD, Waide RB, Scalley TH, et al. Populations are not declining and food webs are not collapsing at the Luquillo Experimental Forest. Proc Natl Acad Sci. 2019:116(25):12143-4.

22. Roskov Y, Kunze T, Paglinawan L, Orrell T, Nicolson D, Culham A, et al. Species 2000 \& ITIS Catalogue of Life, 2013 Annual Checklist. 2013.

23. Stork NE. How many species of insects and other terrestrial arthropods are there on Earth? Annu Rev Entomol. 2018;63:31-45.

24. Schoenly K, Beaver RA, Heumier TA. On the trophic relations of insects: a food-web approach. Am Nat. 1991;137(5):597-638.

25. Klein A-M, Vaissiere BE, Cane JH, Steffan-Dewenter I, Cunningham SA, Kremen C, et al. Importance of pollinators in changing landscapes for world crops. Proc Royal Soc B. 2007;274(1608):303-13.

26. Rader R, Cunningham SA, Howlett BG, Inouye DW. Non-bee insects as visitors and pollinators of crops: biology, ecology and management. Annu Rev Entomol. 2019;65:391-407.

27. Saunders M. Ecosystem services in agriculture: understanding the multifunctional role of invertebrates. Agric For Entomol. 2018;20(2):298-300.

28. Yang LH, Gratton C. Insects as drivers of ecosystem processes. Curr Opin Insect Sci. 2014;2:26-32.

29. Losey JE, Vaughan M. The economic value of ecological services provided by insects. Bioscience. 2006;56(4):311-23.

30. Potts SG, Ngo HT, Biesmeijer JC, Breeze TD, Dicks LV, Garibaldi LA, et al. The assessment report of the Intergovernmental Science-Policy Platform on Biodiversity and Ecosystem Services on pollinators, pollination and food production. 2016

31. Noriega JA, Hortal J, Azcárate FM, Berg MP, Bonada N, Briones MIl, et al. Research trends in ecosystem services provided by insects. Basic Appl Ecol. 2018;26:8-23.

32. Conrad KF, Warren MS, Fox R, Parsons MS, Woiwod IP. Rapid declines of common, widespread British moths provide evidence of an insect biodiversity crisis. Biol Cons. 2006;132(3):279-91.

33. Harris JE, Rodenhouse NL, Holmes RT. Decline in beetle abundance and diversity in an intact temperate forest linked to climate warming. Biol Cons. 2019;240:108219.

34. Forister ML, Pelton EM, Black SH. Declines in insect abundance and diversity: we know enough to act now. Conserv Sci Pract. 2019;1 (8):e80

35. Harvey JA, Heinen R, Armbrecht I, Basset Y, Baxter-Gilbert JH, Bezemer TM, et al. International scientists formulate a roadmap for insect conservation and recovery. Nat Ecol Evol. 2020;4(2):174-6.

36. Cook CN, Carter RWB, Fuller RA, Hockings M. Managers consider multiple lines of evidence important for biodiversity management decisions. J Environ Manage. 2012;113:341-6.

37. O'Leary BC, Woodcock P, Kaiser MJ, Pullin AS. Evidence maps and evidence gaps: evidence review mapping as a method for collating and appraising evidence reviews to inform research and policy. Environ Evid. 2017;6(1):19.

38. Seavy NE, Howell CA. How can we improve information delivery to support conservation and restoration decisions? Biodivers Conserv. 2010;19(5):1261-7.

39. Biedermann R, Achtziger R, Nickel H, Stewart AJA. Conservation of grassland leafhoppers: a brief review. J Insect Conserv. 2005;9(4):229-43.

40. Davies ZG, Tyler C, Stewart GB, Pullin AS. Are current management recommendations for saproxylic invertebrates effective? A systematic review. Biodivers Conserv. 2008:17(1):209-34.

41. Frampton GK, Dorne JLCM. The effects on terrestrial invertebrates of reducing pesticide inputs in arable crop edges: a meta-analysis. J Appl Ecol. 2007:44(2):362-73.

42. Haaland C, Naisbit RE, Bersier L-F. Sown wildflower strips for insect conservation: a review. Insect Conserv Divers. 2011;4(1):60-80.

43. Nicholls $\mathrm{Cl}$, Altieri MA. Plant biodiversity enhances bees and other insect pollinators in agroecosystems. A review. Agron Sustain Dev. 2013;33(2):257-74.

44. Samways MJ. Insect conservation: a synthetic management approach. Annu Rev Entomol. 2007:52:465-87.

45. Stewart AJ, New TR, others. Insect conservation in temperate biomes: issues, progress and prospects. Insect conservation biology. 2007:1-33

46. van Klink R, WallisDeVries MF. Risks and opportunities of trophic rewilding for arthropod communities. Philos Trans Royal Soc B. 2018;373(1761):20170441.

47. Snilstveit B, Vojtkova M, Bhavsar A, Gaarder M. Evidence gap maps—a tool for promoting evidence-informed policy and prioritizing future research: The world bank; 2013.
48. Aromataris E, Fernandez R, Godfrey CM, Holly C, Khalil H, Tungpunkom P. Summarizing systematic reviews: methodological development, conduct and reporting of an umbrella review approach. JBI Evidence Implement. 2015;13(3):132-40.

49. Collins AM, Coughlin D, Randall N. Engaging environmental policy-makers with systematic reviews: challenges, solutions and lessons learned. Environ Evid. 2019;8(1):2

50. Cook CN, Possingham HP, Fuller RA. Contribution of systematic reviews to management decisions. Conserv Biol. 2013;27(5):902-15.

51. Haddaway NR, Macura B, Whaley P, Pullin AS. ROSES RepOrting standards for Systematic Evidence Syntheses: pro forma, flow-diagram and descriptive summary of the plan and conduct of environmental systematic reviews and systematic maps. Environ Evid. 2018;7(1):7.

52. R Core Team. R: A language and environment for statistical computing. Vienna, Austria: R Foundation for Statistical Computing; 2020.

53. Westgate MJ, Grames EM. synthesisr: Import, assemble, and deduplicate bibliographic datasets. R package version 0.3.0 ed2020.

54. Fedrowitz K, Koricheva J, Baker SC, Lindenmayer DB, Palik B, Rosenvald R, et al. Can retention forestry help conserve biodiversity? A meta-analysis. J Appl Ecol. 2014;51(6):1669-79.

55. Hall DM. Insect pollinator conservation policy innovations at subnational levels: lessons for lawmakers. Environ Sci Policy. 2019;93:118-28.

56. Hopwood J, Black SH, Lee-Mäder E, Charlap A, Preston R, Mozumder K, et al. Literature review: pollinator habitat enhancement and best management practices in highway rights-of-way. Washington, DC: The Xerces Society for Invertebrate Conservation and ICF International. Federal Highway Administration, 2015 2015. Report No.

57. Eales J, Haddaway NR, Bernes C, Cooke SJ, Jonsson BG, Kouki J, et al. What is the effect of prescribed burning in temperate and boreal forest on biodiversity, beyond pyrophilous and saproxylic species? A systematic review. Environ Evid. 2018;7(1):19.

58. Gaston KJ, Davies TW, Bennie J, Hopkins J. Reducing the ecological consequences of night-time light pollution: options and developments. J Appl Ecol. 2012;49(6):1256-66.

59. Koshida C, Katayama N. Meta-analysis of the effects of rice-field abandonment on biodiversity in Japan. Conserv Biol. 2018;32(6):1392-402.

60. Tucker $\mathrm{G}$. Review of the impacts of heather and grassland burning in the uplands on soils, hydrology and biodiversity - ENRR550.

61. Winter S, Bauer T, Strauss P, Kratschmer S, Paredes D, Popescu D, et al. Effects of vegetation management intensity on biodiversity and ecosystem services in vineyards: A meta-analysis. J Appl Ecol. 2018;55(5):2484-95.

62. Ellis S, Bourne NA, Bulman CR. Landscape-scale conservation for butterflies and moths: lessons from the UK. Wareham: Butterfly Conservation; 2012. p. 2012.

63. Grames EM, Stillman AN, Tingley MW, Elphic CS. litsearchr: automated search term selection and search strategy for systematic reviews. R package version 0.4.1. ed2020.

64. Grames EM, Stillman AN, Tingley MW, Elphick CS. An automated approach to identifying search terms for systematic reviews using keyword cooccurrence networks. Methods Ecol Evol. 2019;10(10):1645-54.

65. Amano T, González-Varo JP, Sutherland WJ. Languages are still a major barrier to global science. PLoS Biol. 2016;14(12):e2000933.

66. Egger M, Zellweger-Zähner T, Schneider M, Junker C, Lengeler C, Antes $G$. Language bias in randomised controlled trials published in English and German. Lancet. 1997;350(9074):326-9.

67. Grames EM, Montgomery GA, Haddaway NR, Dicks LV, Elphic CS, Matson TA, et al. Trends in global insect abundance and biodiversity: A communitydriven systematic map protocol. Open Science Framework. 2019.

68. Cohen J. Weighted kappa: nominal scale agreement provision for scaled disagreement or partial credit. Psychol Bull. 1968;70(4):213-20.

69. CEE. The Collaboration for Environmental Evidence Synthesis Appraisal Tool (CEESAT). Version 2. The Collaboration for Environmental Evidence; 2018

70. Woodcock P, Pullin AS, Kaiser MJ. Evaluating and improving the reliability of evidence syntheses in conservation and environmental science: a methodology. Biol Cons. 2014;176:54-62.

\section{Publisher's Note}

Springer Nature remains neutral with regard to jurisdictional claims in published maps and institutional affiliations. 